\title{
Interrelations Between Continuous and Discrete Lattice Filter Structures
}

\author{
Steven R. Weller, Arie Feuer, Graham C. Goodwin, and H. Vincent Poor
}

\begin{abstract}
Lattice filter structures have a long history in the filtering and prediction of discrete-time signals. Often these discrete-time signals arise from the sampling of an underlying continuous-time process, and the limiting behavior of the filter as the sampling rate increases is rarely considered. In this paper it is shown that this issue is resolved if the standard formulation of the lattice filter structure, based on the forward shift operator, is replaced by an alternative formulation based on the incremental difference, or delta, operator.

The paper contains two contributions. First, the continuous and discrete lattice algorithms are presented in a unified framework, thereby revealing their common structure. Secondly, it is shown that when the discrete-time signal is obtained by sampling an underlying continuous-time process, the lattice filter corresponding to the discrete case converges, in a well-defined sense, to the solution of the underlying continuous problem as the sampling period approaches zero.
\end{abstract}

\section{INTRODUCTION}

$\mathbf{L}$ ATTICE filter structures have been studied extensively by various authors in the context of the modeling of discretetime signal processes in autoregressive form, e.g, Morf et al. [16], Honig and Messerschmitt [6], and Goodwin and Sin [5]. The lattice form arises by orthogonalizing the past data, replacing this original data by a sequence of orthogonal residuals spanning the same space. Because the lattice filter produces as its output one-step-ahead and one-step-backward residuals of the incoming observations, it is straightforward to use it as a one-step prediction filter [3]. As such, the lattice filter is an alternative to the common transversal filter structure for realizing a restricted complexity one-step predictor (the Levinson predictor).

By considering the one-step-ahead prediction problem as a special case of the more general problem of estimating a smooth signal buried in white noise, we reformulate the lattice filter using an incremental difference operator-the delta operator [15]. In this formulation, the sampling interval appears explicitly, and the discrete lattice structure is presented in a fashion that leads naturally to a continuous form as the sampling rate increases. The continuous lattice filter presented here appears in the work of Kailath et al. [2], [10], [13]. We

Manuscript received November 14, 1991; revised manuscript received June 22, 1992, and February 2, 1993. This paper was recommended by Associate Editor G.S. Moschytz.

S.R. Weller and G.C. Goodwin are with the Centre for Industrial Control science, Department of Electrical and Computer Engineering, University of Newcastle, N.S.W. 2308, Australia.

A. Feuer is with the Technion-Israel Institute of Technology, Dept. of Electrical Engineering, Technion City, Haifa 32000 , Israel.

H.V. Poor is with the Department of Electrical Engineering and Computer Science, Princeton University, Princeton, NJ 08544.

IEEE Log Number 9208428. present the filter here to highlight the connection between the continuous lattice filter and its more common discrete counterpart.

We will also re-examine the one-step-ahead prediction problem, and present an appropriate continuous-time counterpart. This work complements earlier studies by Kailath et al. [2], [10] and Pham and le Breton [12], [17] and presents these results in a unified framework. In the latter work high order derivatives of the process are regressed on lower order derivatives. This leads to an interesting extension of the Levinson-Durbin approach to continuous time. However, due to the nature of the regressors, the Toeplitz nature of the discrete problem is lost, and this means that there is no simple order recursion. Hence the method is not directly analogous to the discrete case and cannot be considered a natural continuous version of the discrete problem. By way of contrast, the procedure we develop is directly analogous to the discrete case and is obtained by a natural limiting process in which the sampling interval goes to zero.

There have been several other recent papers dealing with Levinson type algorithms using fast-sampled data [8], [18]. However, this latter work mirrors the continuous time all-pole work [12], [17] mentioned above. The filter structure presented here differs in two significant ways from the algorithms in [12], [17], [18]. First, in those references, the continuous-time signal process is assumed to be smooth, i.e., the first $n$ mean square derivatives of the process are assumed to exist. Our signal model, on the other hand, is a smooth signal in additive white noise. The presence of this noise necessitates low-pass filtering of the signal prior to sampling. Moreover, it shall be seen that this prefiltering is crucial in obtaining a rapprochement between the discrete-time and continuous-time lattice filtering problems. Secondly, our limiting procedure (in passing from discrete-time to continuous-time) involves considering a fixed time interval, and allowing the number of sample points to increase. By way of contrast, in the work of Vijayan et al. [18], the filter order is fixed as the sampling period tends to zero.

Our development will be based on an extension of the delta operator [15]. This operator is generally superior to the shift operator when fast sampling is employed. For example, if one uses AR models, then it can be shown that the delta form is numerically superior to the shift form at high sampling rates [4], [18]. The essential reason is that, in the shift form, the filter coefficients converge to the binomial coefficients independently of the underlying continuous-time process. Thus numerical precision is lost due to the fact that the true system 
information is encoded in the last significant bits of the AR coefficients. However, a different phenomenon occurs in the case of lattice filters. For the latter problem, we show below that the coefficients in the normal shift form are $\mathcal{O}(\Delta)$. Thus some form of scaling is necessary to alleviate problems due to coefficient quantization. The delta formulation used here has the advantage of making this scaling explicit. Also, this formulation is pivotal in establishing the connection with the underlying continuous-time filter.

The layout of the paper is as follows. In Section II, we review the structure and some features of the discrete lattice filter. We show how such a filter can be used for prediction purposes, and indicate its close relationship with the Levinson predictor. The signal process we observe is taken to be a signal $(S)$ in white measurement noise $(V)$, and the prediction problem is referred to as the "discrete time $S V$ problem." Note that the problem of predicting a signal from past measurements of itself is a special case of the $S V$ problem. In Section III, the corresponding continuous time $S V$ problem is proposed, and we outline its solution. Sections II and III serve to present the continuous and discrete $S V$ problems within a common framework. In Section IV, the link between the continuous and discrete $S V$ problems of Sections II and III is made apparent by presenting the discrete-time structure using a divided difference (delta) operator. In Section V, we show a different facet of the connection between the continuous and discrete $S V$ problems by obtaining the discrete solution when an underlying continuous process is sampled. It is shown that the discrete-time problem converges to the continuous-time problem in a well-defined fashion, and that the corresponding filters are intimately linked.

Since we will need to deal with both continuous time and sampled signals, we introduce the following notation:

- $t$ denotes real time (measured, for example, in seconds),

- $\Delta$ denotes the sampling period $(\Delta=0$ signifies continuous time),

- $i, j, k, l, m, n \in \mathbb{Z}$.

For a sampled signal, we will be interested only in those times $t$ which can be expressed as $t=k \Delta$ for some $k \in \mathbb{Z}$. In the discrete time case we will often suppress the explicit dependence on $\Delta$ by writing $\bar{y}(k)=y(k \Delta)$, where $y: \mathbf{R} \rightarrow \mathbf{R}$.

\section{LATTICE FILTERS AND THE SV PROBLEM IN SHIFTFORM}

In this section, the essential features of the lattice filter structure introduced by Itakura and Saito [7] are reviewed. The key feature of the lattice filter is that it is an orthogonalization device which replaces the original measurement process by a sequence of orthogonal residuals spanning the same space. It shall be seen that the production of these residuals permits a slight extension of the basic lattice structure, by which onestep-ahead and one-step-backward predictors for the signal process are readily obtained.

Suppose the measurement process $\{\bar{y}(\cdot)\}$ consists of a signal process $\{\bar{s}(\cdot)\}$ (with known covariance structure) corrupted by white measurement noise $\{\bar{v}(\cdot)\}$. Predicting subsequent values of the signal process based on measurements of $\{\bar{y}(\cdot)\}$ will be referred to as the discrete-time " $S V$ problem." It is in this context that the lattice filter shall be presented. In the sequel we shall rely on projection arguments using the usual notion of a Hilbert space spanned by a set of random variables (see, e.g., [14]).

Consider a real-valued, wide-sense stationary discretetime random process $\{\bar{y}(\cdot)\}$. Let $H$ be the Hilbert space spanned by the random process $\{\bar{y}(\cdot)\}$, with inner product $\langle\bar{y}(k), \bar{y}(j)\rangle \triangleq \mathcal{E}\{\tilde{y}(k) \bar{y}(j)\}$, where $\mathcal{E}\{\cdot\}$ denotes the expectation operator. We denote by $\bar{Y}_{j}^{j+k}$ for $k \geq 0$ the closed, linear subspace of $H$ spanned by $\{\bar{y}(j), \bar{y}(j+1), \cdots, \bar{y}(j+$ $k)\} . \bar{Y}_{j}^{j+k}$ for $k<0$ denotes the empty space. For every $\bar{y}(i) \in H, \hat{\bar{y}}\left(i \mid \bar{Y}_{j}^{j+k}\right)$ denotes the orthogonal projection of $\bar{y}(i)$ onto $\bar{Y}_{j}^{j+k}$; that is,

$$
\begin{aligned}
\mathcal{E}\left\{\left(\bar{y}(i)-\hat{\bar{y}}\left(i \mid \bar{Y}_{j}^{j+k}\right)\right) \bar{y}(l)\right\}=0, & \\
& \quad l=j, j+1, \cdots, j+k
\end{aligned}
$$

Thus $\hat{\bar{y}}\left(i \mid \bar{Y}_{j}^{j+k}\right)$ is the wide-sense conditional expectation of $\bar{y}(i)$ given the observations $\bar{Y}_{j}^{j+k}$.

\section{Assumptions}

D1: The processes $\{\bar{y}(\cdot)\}$ and $\{\bar{s}(\cdot)\}$ are related by

$$
\bar{y}(k)=\bar{s}(k)+\bar{v}(k), \quad \forall k \in \mathbf{Z} .
$$

D2: The process $\{\bar{s}(\cdot)\}$ is zero mean and wide-sense stationary with known covariance function $\bar{W}_{i}$. The process $\{\bar{v}(\cdot)\}$ is zero mean and white with variance $\bar{\gamma}$, and $\{\bar{s}(\cdot)\},\{\bar{v}(\cdot)\}$ are uncorrelated, i.e.,

$$
\begin{aligned}
\mathcal{E}\{\bar{s}(k) \bar{s}(k+i)\} & =\bar{W}_{i}, \quad \forall k, i \in \mathbb{Z} \\
\mathcal{E}\{\bar{v}(k) \bar{v}(j)\} & =\bar{\gamma} \delta_{k-j}, \quad \forall k, j \in \mathbf{Z} \\
\mathcal{E}\{\bar{s}(k) \bar{v}(j)\} & =0, \quad \forall k, j \in \mathbf{Z} .
\end{aligned}
$$

Definition 2.1 ( $S V$ problem in discrete time): Let processes $\{\bar{y}(\cdot)\},\{\bar{s}(\cdot)\}$ and $\{\bar{v}(\cdot)\}$ satisfy assumptions D1, D2. Then predicting the value of the signal process $\{\bar{s}(\cdot)\}$ at a specific time $k$, say, given a finite measurement record $\{\bar{y}(k-$ $N), \cdots, \bar{y}(k-1)\}$, say, will be referred to as the SV problem in discrete time.

Let $\hat{\bar{s}}\left(k \mid \bar{Y}_{k-N}^{k-1}\right)$ denote the orthogonal projection of $\bar{s}(k)$ onto the linear subspace $\bar{Y}_{k-N}^{k-1}$. Thus $\hat{\bar{s}}\left(k \mid \bar{Y}_{k-N}^{k-1}\right)$ is the optimal linear least squares predictor of $\bar{s}(k)$ given the $N$ previous observations $\{\bar{y}(k-N), \cdots, \bar{y}(k-1)\}$. The forward residual of order $N$ is defined as follows:

$$
\bar{e}_{f}(N, k) \triangleq \bar{y}(k)-\hat{\bar{s}}\left(k \mid \bar{Y}_{k-N}^{k-1}\right) .
$$

Similarly the backward residual is defined

$$
\bar{e}_{b}(N, k) \triangleq \bar{y}(k-N)-\hat{\bar{s}}\left(k-N \mid \bar{Y}_{k-N+1}^{k}\right)
$$

where

$$
\bar{e}_{f}(0, k)=\bar{e}_{b}(0, k)=\bar{y}(k) .
$$




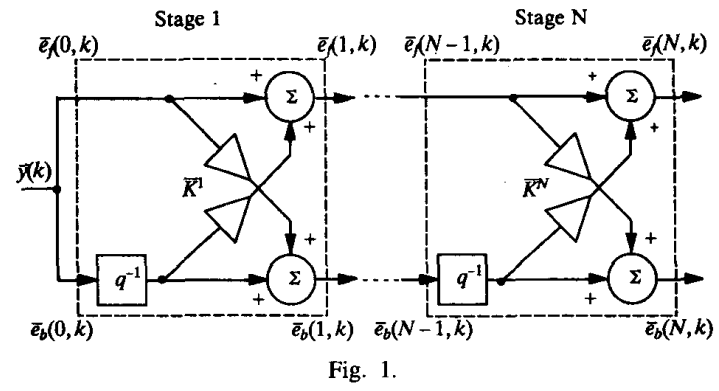

It can be shown (e.g., [5, p. 285]) that the forward and backward residuals satisfy the order recursive formulas

$$
\begin{aligned}
& \dot{\bar{e}}_{f}(N+1, k)=\bar{e}_{f}(N, k)+\bar{K}^{N+1} \bar{e}_{b}(N, k-1) \\
& \bar{e}_{b}(N+1, k)=\bar{e}_{b}(N, k-1)+\bar{K}^{N+1} \bar{e}_{f}(N, k)
\end{aligned}
$$

comprising the lattice structure depicted in Fig. 1, where $\bar{K}^{i}$ are referred to as a reflection coefficients.

The forward and backward residuals satisfy the following orthogonality properties [5]:

$$
\begin{aligned}
\mathcal{E}\left\{\bar{e}_{f}(k, i+k) \bar{e}_{f}(i, i+j)\right\} & =\bar{R}^{k} \delta_{j-k} \\
\mathcal{E}\left\{\bar{e}_{b}(k, i) \bar{e}_{b}(j, i)\right\} & =\bar{R}^{k} \delta_{j-k} .
\end{aligned}
$$

It follows from (7) and (8) and Definitions (2) and (3) that both $\left\{\bar{e}_{b}(0, k), \bar{e}_{b}(1, k), \cdots, \bar{e}_{b}(N-1, k)\right\}$ and $\left\{\bar{e}_{f}(0, k-\right.$ $\left.N+1), \bar{e}_{f}(1, k-N+2), \cdots, \bar{e}_{f}(N-1, k)\right\}$ are orthogonal bases for $\bar{Y}_{k-N+1}^{k}$. Using $\bar{e}_{b}(0, k), \bar{e}_{b}(1, k), \ldots, \bar{e}_{b}(N-1, k)$ as an orthogonal basis for $\bar{Y}_{k-N+1}^{k}$, it can readily be shown (see, e.g. [3]) that a one-step-ahead predictor can be obtained as

$$
\hat{\bar{s}}\left(k+1 \mid \bar{Y}_{k-N+1}^{k}\right)=-\sum_{i=1}^{N} \bar{K}^{i} \bar{e}_{b}(i-1, k) .
$$

Likewise, a corresponding expression for the one step backward predictor can be obtained:

$$
\hat{\bar{s}}\left(k-N \mid \bar{Y}_{k-N+1}^{k}\right)=-\sum_{i=1}^{N} \bar{K}^{i} \bar{e}_{f}(i-1, k-N+1) .
$$

The one-step-ahead predictor in (9) expresses predictions as a weighted sum of backward residuals. It is also possible to form predictions as a weighted sum of past values of the measurement process:

$$
\hat{s}\left(k+1 \mid \bar{Y}_{k-N+1}^{k}\right)=-\sum_{i=1}^{N} \bar{a}_{i}^{N} \bar{y}(k-i+1) .
$$

This is the Levinson predictor, in which the coefficients $\bar{a}_{i}^{N}$ are chosen to minimize the mean square prediction error

$$
\mathcal{E}\left\{\left(\bar{s}(k+1)-\hat{s}\left(k+1 \mid \bar{Y}_{k-N+1}^{k}\right)\right)^{2}\right\}
$$

and are obtained as the solution of the Yule-Walker equations

$$
\left[\begin{array}{cccc}
\left(\bar{\gamma}+\bar{W}_{0}\right) & \bar{W}_{1} & \cdots & \bar{W}_{N-1} \\
\bar{W}_{1} & \ddots & & \vdots \\
\vdots & & & \bar{W}_{1} \\
\bar{W}_{N-1} & \cdots & & \left(\bar{\gamma}+\bar{W}_{0}\right)
\end{array}\right]\left[\begin{array}{c}
\bar{a}_{1}^{N} \\
\bar{a}_{2}^{N} \\
\vdots \\
\bar{a}_{N}^{N}
\end{array}\right]=-\left[\begin{array}{c}
\bar{W}_{1} \\
\bar{W}_{2} \\
\vdots \\
\bar{W}_{N}
\end{array}\right] .
$$

In practice, (12) is usually solved recursively in $N$ by the Levinson algorithm [11], which exploits the Toeplitz structure of the matrix on the left-hand side.

The following well-known relationship also holds.

Lemma 2.1: The coefficient $\bar{a}_{N}^{N}$ in the Levinson predictor (11) is identical to the reflection coefficient $\bar{K}^{N}$ in the lattice filter.

Proof: See [5, p. 291].

\section{The SV PRoBlem IN CONTINUOUS-TIME}

In this section, we consider the continuous time analog of the discrete time $S V$ problem introduced in Section II. That is, we consider the problem of estimating the value of a continuous time signal process $\{s(\cdot)\}$ at time $t$ based on measurements of a related process $\{y(\cdot)\}$ over a finite observation window of length $T$. Such a problem has been considered previous; see, for example, Kailath [10].

\section{Assumptions}

C1: The processes $\{y(\cdot)\}$ and $\{s(\cdot)\}$ are related by

$$
y(\tau)=s(\tau)+v(\tau), \quad \forall \tau \in \mathbb{R} .
$$

C2: The process $\{s(\cdot)\}$ is zero mean and wide-sense stationary with known covariance function $W(\cdot),\{v(\cdot)\}$ is a zero mean "white noise" process with incremental covariance $\Gamma d \tau$, and $\{s(\cdot)\},\{v(\cdot)\}$ are uncorrelated, i.e.,

$$
\begin{aligned}
\mathcal{E}\{s(t) s(t-\tau)\} & =W(\tau), \quad \forall t, \tau \in \mathbb{R} \\
\mathcal{E}\{v(t) v(\tau)\} & =\Gamma \delta(t-\tau), \quad \forall t, \tau \in \mathbb{R} \\
\mathcal{E}\{s(t) v(\tau)\} & =0, \quad \forall t, \tau \in \mathbb{R} .
\end{aligned}
$$

Definition 3.1 ( $S V$ problem in continuous time):

Let processes $\{y(\cdot)\},\{s(\cdot)\}$, and $\{v(\cdot)\}$ satisfy assumptions $\mathrm{C} 1, \mathrm{C} 2$. Then predicting the value of the signal $\{s(\cdot)\}$ at a specific time $t$, say, given a finite measurement record $\{y(\tau): t-T \leq \tau \leq t\}$, say, will be referred to as the $S V$ problem in continuous time.

Motivated by the results of the previous section, our forward estimate of $s(t)$ based on measurements $\{y(\tau): t-T \leq \tau \leq t\}$ will be denoted $\hat{s}\left(t \mid Y_{t-T}^{t}\right)$, and will take the form

$$
\hat{s}\left(t \mid Y_{t-T}^{t}\right)=-\int_{t-T}^{t} A(T, t-\sigma) y(\sigma) d \sigma
$$

where $A(T, \cdot)$ is a continuous function with support in $[0, T]$. By a slight abuse of terminology, $A$ will be called the filter. Similarly, the backwards estimate is assumed to take the form

$$
\hat{s}\left(t-T \mid Y_{t-T}^{t}\right)=-\int_{t-T}^{t} B(T, t-\sigma) y(\sigma) d \sigma .
$$


The following results summarizes some of the properties of the estimators given above.

Lemma 3.1: Let $\hat{s}\left(t \mid Y_{t-T}^{t}\right)$ and $\hat{s}\left(t-T \mid Y_{t-T}^{t}\right)$ be as given in (13) and (14). Let the forward and backward residuals be defined by

$$
\begin{aligned}
& e_{f}(T, t) \triangleq y(t)-\hat{s}\left(t \mid Y_{t-T}^{t}\right) \\
& e_{b}(T, t) \triangleq y(t-T)-\hat{s}\left(t-T \mid Y_{t-T}^{t}\right) .
\end{aligned}
$$

then

1) $A(T, \cdot)$ satisfies the following integral equation [9]:

$$
\begin{aligned}
& W(\tau)+\Gamma A(T, \tau)+\int_{0}^{T} A(T, \sigma) W(\tau-\sigma) d \sigma=0, \\
& 0 \leq \tau \leq T .
\end{aligned}
$$

2) $B(T, \cdot)$ is the time-reverse of $A(T, \cdot)$ :

$$
B(T, T-\sigma)=A(T, \sigma), \quad 0 \leq \sigma \leq T .
$$

3) $A(T, \cdot)$ satisfies the following equation, which is known as the Bellman-Krein-Siegert relation [13]:

$$
\frac{\partial A(T, \sigma)}{\partial T}=A(T, T-\sigma) A(T, T), \quad 0 \leq \sigma \leq T .
$$

4) The forward and backward residuals satisfy the following relationship termed the continuous time lattice structure:

$$
\frac{\partial}{\partial T}\left[\begin{array}{l}
e_{f}(T, t) \\
e_{b}(T, t)
\end{array}\right]=\left[\begin{array}{cc}
0 & A(T, T) \\
A(T, T) & -\frac{\partial}{\partial t}
\end{array}\right]\left[\begin{array}{l}
e_{f}(T, t) \\
e_{b}(T, t)
\end{array}\right]
$$

5) Forward and backward estimates can be expressed in terms of prediction residuals:

$$
\begin{aligned}
\hat{s}\left(t \mid Y_{t-T}^{t}\right) & =-\int_{0}^{T} A(\tau, \tau) e_{b}(\tau, t) d \tau \\
\hat{s}\left(t-T \mid Y_{t-T}^{t}\right) & =-\int_{0}^{T} A(\tau, \tau) e_{f}(\tau, t) d \tau .
\end{aligned}
$$

6) $\left\{e_{b}(\cdot, \cdot)\right\}$ and $\left\{e_{f}(\cdot, \cdot)\right\}$ satisfy the following orthogonality properties:

$$
\begin{aligned}
\mathcal{E}\left\{e_{b}(T, t) e_{b}(T-\tau, t)\right\} & =0, & & \tau>0 \\
\mathcal{E}\left\{e_{f}(T, t) e_{f}(T-\tau, t-\tau)\right\} & =0, & & \tau>0
\end{aligned}
$$

Proof: There does not appear to be a reference where these facts are all brought together in a unified way. Therefore, for completeness, we briefly establish them in Appendix A. $\square$

The above Lemma summarizes the key properties needed in the subsequent development. However many other properties hold. For example, the operators $A$ and $B$ also satisfy the following Krein-Levinson equations [10]:

$$
\begin{aligned}
\left(\frac{\partial}{\partial T}+\frac{\partial}{\partial t}\right) B(T, t) & =A(T, T) A(T, t) \\
\frac{\partial}{\partial T} A(T, t) & =A(T, T) B(T, t) .
\end{aligned}
$$

\section{Discrete-TIME SV Problem IN DELTA Form}

In Lemma 3.1, the relationship (20) was proved and termed the continuous time lattice structure. In Section II, the discrete time prediction residuals $\bar{e}_{f}(N, k)$ and $\bar{e}_{b}(N, k)$ were shown to satisfy the recursions (5) and (6). However, the discretetime and continuous-time lattice structures (20) and (5) and (6) bear little resemblance to one another. We show below that by recasting the discrete-time solution in difference form, the connection is clarified.

The delta operator is a divided difference operator proposed in [15] as the appropriate discrete time analog of the differentiation operator:

$$
\delta \triangleq \frac{q-1}{\Delta}
$$

where $q$ is the conventional forward shift operator, and $\Delta$ is the sampling interval. Thus

$$
\delta x(k)=\frac{x(k+1)-x(k)}{\Delta} .
$$

Here we extend the delta operator to functions of multiple independent variables.

$$
\begin{aligned}
& \delta^{i} f\left(k_{1}, \cdots, k_{i}, \cdots, k_{N}\right) \\
& \triangleq \frac{f\left(k_{1}, \cdots, k_{i}+1, \cdots, k_{N}\right)-f\left(k_{1}, \cdots, k_{i}, \cdots, k_{N}\right)}{\Delta} \\
& 1 \leq i \leq N
\end{aligned}
$$

Thus $\delta^{i} f$ is a discrete time analog of partial differentiation with respect to the $i$ th argument of the function $f$.

With this notation in mind, we can rewrite (5) and (6) as

$$
\begin{aligned}
\frac{\bar{e}_{f}(N+1, k)-\bar{e}_{f}(N, k)}{\Delta}= & \frac{\bar{K}^{N+1}}{\Delta} \bar{e}_{b}(N, k-1) \\
\frac{\bar{e}_{b}(N+1, k)-\bar{e}_{b}(N, k)}{\Delta}= & \frac{\bar{e}_{b}(N, k-1)-\bar{e}_{b}(N, k)}{\Delta} \\
& +\frac{\bar{K}^{N+1}}{\Delta} \bar{e}_{f}(N, k) .
\end{aligned}
$$

If we also introduce the definition

$$
\bar{A}(N, i) \triangleq \frac{\bar{a}_{i}^{N}}{\Delta}, \quad i=1,2, \cdots, N
$$

then from Lemma 2.1,

$$
\bar{A}(N+1, N+1)=\frac{\bar{K}^{N+1}}{\Delta} .
$$

With (32) and the notation introduced in (28), Equations (29) and (30) may be written

$$
\begin{aligned}
\delta^{1} & {\left[\begin{array}{l}
\bar{e}_{f}(N, k) \\
\bar{e}_{b}(N, k)
\end{array}\right] } \\
= & {\left[\begin{array}{cc}
0 & \bar{A}(N+1, N+1) \\
\bar{A}(N+1, N+1) & -\delta^{2}
\end{array}\right] } \\
& \cdot\left[\begin{array}{c}
\bar{e}_{f}(N, k) \\
\bar{e}_{b}(N, k-1)
\end{array}\right] .
\end{aligned}
$$


Also, substituting definition (31) into the one step forward and backward predictions in the discrete time $S V$ problem (9), (10) gives

$$
\begin{aligned}
\hat{\bar{s}}\left(k+1 \mid \bar{Y}_{k-N+1}^{k}\right) & =-\Delta \sum_{i=1}^{N} \bar{A}(i, i) \bar{e}_{b}(i-1, k) \\
\hat{\bar{s}}\left(k-N \mid \bar{Y}_{k-N+1}^{k}\right) & =-\Delta \sum_{i=1}^{N} \bar{A}(i, i) \bar{e}_{f}(i-1, k-N+1)
\end{aligned}
$$

while the Levinson predictor of (11) becomes

$$
\hat{\bar{s}}\left(k+1 \mid \bar{Y}_{k-N+1}^{k}\right)=-\Delta \sum_{i=1}^{N} \bar{A}(N, i) \bar{y}(k-i+1) .
$$

Comparison of (13), (20)-(22), and (33)-(36) reveals the intimate connection between the continuous and discrete lattice filters. In the next section, we shall investigate the nature of this connection in more quantitative terms.

\section{SAMPLING AN UNDERLYING CONTINUOUS-TIME PROCESS}

In the previous section, it was shown that the form of the continuous-time and discrete-time time lattice structures are intimately related. In this section, we investigate this relationship more closely by quantitatively examining the limit (as the sampling period tends to zero) of the discrete-time filter applied to a sampled form of an underlying continuous-time process.

Consider a signal $\{y(\cdot)\}$ satisfying assumptions $\mathrm{C} 1, \mathrm{C} 2$. Note that it makes no sense to directly sample $\{y(\cdot)\}$ since this would lead to a discrete-time process of unbounded variance. This problem is resolved by replacing the impractical ideal sampler by an ideal sampler incorporating an anti-aliasing filter before the sampling process. The filter we use here is a "reset and integrate" type (see, e.g., [15, p. 279]), so that

$$
\bar{y}(k+1)=\frac{1}{\Delta} \int_{k \Delta}^{(k+1) \Delta} y(t) d t .
$$

The following Lemma establishes statistical properties of the sampled signal.

Lemma 5.1: Let $\{y(\cdot)\}$ satisfying assumptions $\mathrm{C} 1, \mathrm{C} 2$ be filtered prior to sampling as in (37). Then the resulting discrete time process $\{\bar{y}(\cdot)\}$ satisfies assumptions D1, D2, where

$$
\begin{aligned}
\bar{\gamma} & =\frac{\Gamma}{\Delta} \\
\bar{W}_{j-k} & =\frac{1}{\Delta^{2}} \int_{(j-1) \Delta}^{j \Delta} \int_{(k-1) \Delta}^{k \Delta} W\left(t_{2}-t_{1}\right) d t_{2} d t_{1} .
\end{aligned}
$$

Proof: That D1 is satisfied follows directly by substitution of $y(\tau)=s(\tau)+v(\tau)$ into (37).

$$
\begin{aligned}
& \mathcal{E}\{\bar{s}(j) \bar{s}(k)\} \\
& \quad=\mathcal{E}\left\{\frac{1}{\Delta} \int_{(j-1) \Delta}^{j \Delta} s(t) d t \cdot \frac{1}{\Delta} \int_{(k-1) \Delta}^{k \Delta} s(t) d t\right\}
\end{aligned}
$$

$$
=\frac{1}{\Delta^{2}} \int_{(j-1) \Delta}^{j \Delta} \int_{(k-1) \Delta}^{k \Delta} \mathcal{E}\left\{s\left(t_{1}\right) s\left(t_{2}\right)\right\} d t_{2} d t_{1}
$$

from which (39) follows immediately.

$$
\begin{aligned}
\mathcal{E}\{\bar{v}(j) \bar{v}(k)\} & =\frac{1}{\Delta^{2}} \int_{(k-1) \Delta}^{k \Delta} \int_{(k-1) \Delta}^{k \Delta} \mathcal{E}\{v(t) v(\tau)\} d t d \tau \\
& =\frac{\Gamma}{\Delta^{2}} \int_{(k-1) \Delta}^{k \Delta} \int_{(j-1) \Delta}^{j \Delta} \delta(t-\tau) d t d \tau \\
& =\frac{\Gamma}{\Delta} \delta_{k-j} .
\end{aligned}
$$

That $\{\bar{s}(\cdot)\}$ and $\{\bar{v}(\cdot)\}$ are uncorrelated is apparent from the uncorrelatedness of $\{s(\cdot)\}$ and $\{v(\cdot)\}$.

Given the continuous-time process and its sampled version $\{\bar{y}(\cdot)\}$ as in (37), our intention now is to establish the connection between the function $A(T, \cdot):[0, T] \rightarrow \mathbb{R}$ defining the continuous-time filter (13) and the coefficients $\{\bar{A}(N, i)\}_{i=1}^{N}$ defining the discrete-time filter (36). Recall from Lemma 3.1 that $A(T, \cdot)$ satisfies the integral equation (17) where $W(\cdot)$ is the covariance function of the signal process $\{s(\cdot)\}$ (assumed known, continuous, and bounded), $A(T, \cdot)$ is the required continuous solution function, and $0<\Gamma<\infty$.

Remark 5.1: Equation (17) is a Fredholm integral equation of the second kind, with kernel $W(\cdot)$. A well-known sufficient condition for the existence of a unique solution $A(T, \cdot)$ which is continuous on $[0, T]$ is that

$$
\Gamma>W(0) T
$$

where $W(0) \equiv \sup _{\sigma \in \mathbf{R}} W(\sigma)$. In the sequel, we shall assume (40) is satisfied.

The coefficients $\{\bar{A}(N, i)\}_{i=1}^{N}$ of the discrete-time filter (36) are given by (31) where $\bar{a}_{i}^{N}$ comprise the solution of the Yule-Walker equations (12). We aim to show that as $\Delta \rightarrow 0$, the discrete-time coefficients $\{A(N, i)\}_{i=1}^{N}$ converge to sample values of the continuous time filter $A(T, \cdot)$ satisfying (17). To this end, we will construct a continuous-time, piecewiseconstant function (denoted $\bar{A}^{\Delta}$ ) from $\{\bar{A}(N, i)\}_{i=1}^{N}$ and show that $\bar{A}^{\Delta} \rightarrow A$ on $[0, T]$ as $\Delta \rightarrow 0$.

In all subsequent working, we assume that $T>0$ is fixed, and that for any $N \in \mathbb{Z}_{+}, \Delta$ is such that

$$
T=N \Delta \text {. }
$$

The proposed scheme is reminiscent of a numerical solution for integral equations of the second kind, a problem which has been well studied (e.g., [1, p. 156]). Namely, the kernel $W$ and free term $-W / \Gamma$ in (17) are replaced by approximations $W^{*}$ and $-W^{*} / \Gamma$, respectively, and a bound is obtained for the error between the solution $A$ of (17) and $A^{*}$ of

$$
A^{*}(T, \tau)=-\frac{W^{*}(\tau)}{\Gamma}-\frac{1}{\Gamma} \int_{0}^{T} A^{*}(T, \sigma) W^{*}(\tau-\sigma) d \sigma
$$

In the method below, however, the approximate solution $\bar{A}^{\Delta}$ is constructed from the Levinson coefficients $\left\{\bar{a}_{i}^{N}\right\}_{i=1}^{N}$, and an appropriate $W^{\Delta}$ is devised such that the resulting integral equation is similar in form to (17) (cf., Definition 5.1 and Lemma 5.2 below). 
Definition 5.1: $\bar{A}^{\Delta}(\cdot)$ and $W^{\Delta}(\cdot)$ are defined as follows:

$$
\begin{aligned}
& \bar{A}^{\Delta}(\tau) \triangleq\left\{\begin{array}{l}
\bar{A}(N, i), \quad \tau \in[(i-1) \Delta, i \Delta), \\
i=1,2, \cdots, N-1 \\
\bar{A}(N, N), \quad \tau \in[(N-1) \Delta, T]
\end{array}\right. \\
& W^{\Delta}(\tau) \triangleq \bar{W}_{i+1}, \quad \tau \in(i \Delta,(i+1) \Delta], \\
& i=-N, \cdots, N-1 \text {. }
\end{aligned}
$$

Using these definitions of $\bar{A}^{\Delta}$ and $W^{\Delta}$, it is possible to formulate an integral equation similar in form to (17) which, when evaluated at particular points in $[0, T]$, is equivalent to the Yule-Walker equations (12) defining $\bar{a}_{i}^{N}$. This is established in the following Lemma.

Lemma 5.2: Let $\bar{A}^{\Delta}$ and $W^{\Delta}$ be defined as in (42) and (43). Then for $N, \Delta$ and $T$ satisfying (41), the integral equation

$$
\begin{aligned}
\Gamma \bar{A}^{\Delta}(\tau)+\int_{0}^{T} \bar{A}^{\Delta}(\sigma) W^{\Delta}(\tau-\sigma) d \sigma \\
=-W^{\Delta}(\tau+\Delta), \quad 0 \leq \tau \leq T-\Delta
\end{aligned}
$$

evaluated at $\tau=i \Delta, i=0,1, \cdots, N-1$ is equivalent to the $(i+1)$ th row of $(12)$.

Proof: By substituting $\tau=i \Delta$ for $i=0,1, \cdots, N-1$ into (44), and by using (38) and (31) and Definitions (42) and (43).

The continuous-time and discrete-time filters are now in a compatible form, namely (17) and (44) evaluated at $\tau \in$ $\{0, \Delta, \cdots,(N-1) \Delta\}$. For notational simplicity, the explicit dependence of both $A$ and $\bar{A}^{\Delta}$ on $T$ will subsequently be dropped.

Using (17) and (44), it is possible to compare the behavior of $\bar{A}^{\Delta}$ at the points $\tau=0, \Delta, \cdots,(N-1) \Delta$ with the solution $A(\tau)$ of (17) as $N \rightarrow \infty$ (equivalently, as $\Delta \rightarrow 0$ ). We shall measure the magnitude of a function by its sup-norm, viz.,

$$
\|f\|_{\infty}=\sup _{\sigma \in[0, T]}|f(\sigma)| .
$$

With the above as background, we next present the main result of the paper, in which convergence of the discrete-time filter to the continuous-time filter is established.

Theorem 5.1: Let the continuous-time filter $A(\tau)$ satisfy (17), let the Yule-Walker equations for the discrete-time filter coefficients be represented in integral form by (44), and let $N, \Delta, T$ be such that (41) is satisfied. Then if condition (40) guaranteeing existence and uniqueness of the solution of (17) is satisfied, we may conclude

$$
\left\|A(\tau)-\bar{A}^{\Delta}(\tau)\right\|_{\infty} \rightarrow 0, \quad \text { as } N \rightarrow \infty
$$

for $\tau \in\{0, \Delta, 2 \Delta, \cdots,(N-1) \Delta\}$.
Proof: From (44) and (17),

$$
\begin{aligned}
\| A(\tau) & -\bar{A}^{\Delta}(\tau) \|_{\infty} \\
\leq & \frac{1}{\Gamma}\left\|W^{\Delta}(\tau+\Delta)-W(\tau)\right\|_{\infty} \\
& +\frac{1}{\Gamma} \int_{0}^{T}\|A(\sigma)\|_{\infty}\left\|W^{\Delta}(\tau-\sigma)-W(\tau-\sigma)\right\|_{\infty} d \sigma \\
& +\frac{1}{\Gamma} \int_{0}^{T}\left\|\bar{A}^{\Delta}(\sigma)-A(\sigma)\right\|_{\infty}\left\|W^{\Delta}(\tau-\sigma)\right\|_{\infty} d \sigma .
\end{aligned}
$$

Consider in turn each of the terms on the right-hand side of (45). Using the continuity of $W$, the definition (43) of $W^{\Delta}$, and the definition of $\bar{W}_{j-k}$ from $W$ (cf., 39)), it is straightforward to show that for $\tau \in\{0, \Delta, \cdots,(N-1) \Delta\}$,

$$
\frac{1}{\Gamma}\left\|W^{\Delta}(\tau+\Delta)-W(\tau)\right\|_{\infty}=\mathcal{O}(\Delta) .
$$

Now $A$ is continuous on $[0, T]$; hence bounded. Thus

$$
\frac{1}{\Gamma} \int_{0}^{T}\|A(\sigma)\|_{\infty}\left\|W^{\Delta}(\tau-\sigma)-W(\tau-\sigma)\right\|_{\infty} d \sigma=\mathcal{O}(\Delta)
$$

by the same line of reasoning which lead to (46). Also,

$$
\begin{aligned}
\frac{1}{\Gamma} \int_{0}^{T}\left\|\bar{A}^{\Delta}(\sigma)-A(\sigma)\right\|_{\infty} & \left\|W^{\Delta}(\tau-\sigma)\right\|_{\infty} d \sigma \\
& \leq W(0) T\left\|\bar{A}^{\Delta}(\sigma)-A(\sigma)\right\|_{\infty}
\end{aligned}
$$

for $\sigma \in\{0, \Delta, \cdots,(N-1) \Delta\}$. Combining (45)-(48) gives

$$
\begin{aligned}
\left\|A(\tau)-\bar{A}^{\Delta}(\tau)\right\|_{\infty} \\
\quad \leq \frac{W(0) T}{\Gamma}\left\|A(\tau)-\bar{A}^{\Delta}(\tau)\right\|_{\infty}+\mathcal{O}(\Delta)
\end{aligned}
$$

for $\tau \in\{0, \Delta, \cdots,(N-1) \Delta\}$. But from the condition for existence and uniqueness of the solution $A$ of (17), (cf., Remark 5.1),

$$
\frac{W(0) T}{\Gamma}<1
$$

and so in the limit as $N \rightarrow \infty$ (equivalently, $\Delta \rightarrow 0$ ), (49) can only be true if

$$
\lim _{N \rightarrow \infty}\left\|A(\tau)-\bar{A}^{\Delta}(\tau)\right\|_{\infty}=0
$$

\section{CONCLUSION}

This paper has explored the connection between continuous and discrete lattice filtering algorithms. It has been shown that, when formulated appropriately, there is an intimate connection between the discrete and continuous cases. Further, it has been shown that when the discrete filtering problem arises by sampling an underlying continuous-time signal process, the discrete lattice filter converges to the appropriate continuoustime filter as the sampling period approaches zero. This gives insight into both cases and also indicates the appropriate 
coefficient scaling mechanisms so that the discrete problem is well formulated at fast sampling rates.

We have treated only the case of a scalar measurement process but the extension to vector measurements would seem to follow analogously. Far less obvious extensions may also exist for nonlinear filtering problems. These issues are the subject of on-going research.

VII. APPENDIX A

PROOF OF LEMMA 3.1

Proof: 1) By change of variable in (13),

$$
\hat{s}\left(t \mid Y_{t-T}^{t}\right)=-\int_{0}^{T} A(T, \sigma) y(t-\sigma) d \sigma .
$$

Now by the orthogonality principle,

$$
\mathcal{E}\left\{\left(s(t)-\hat{s}\left(t \mid Y_{t-T}^{t}\right)\right) y(\tau)\right\}=0, \quad t-T \leq \tau \leq t
$$

where

$$
\mathcal{E}\{s(t) y(\tau)\}=W(t-\tau)
$$

and

$$
\begin{aligned}
& \mathcal{E}\left\{\hat{s}\left(t \mid Y_{t-T}^{t}\right) y(\tau)\right\} \\
& \quad=\mathcal{E}\left\{-\int_{0}^{T} A(T, \sigma) y(t-\sigma) d \sigma[s(\tau)+v(\tau)]\right\} \\
& \quad=-\int_{0}^{T} A(T, \sigma)[W(t-\sigma-\tau)+\Gamma \delta(t-\sigma-\tau)] d \sigma .
\end{aligned}
$$

Thus

$$
\begin{aligned}
& W(t-\tau)+\Gamma A(T, t-\tau) \\
& +\int_{0}^{T} A(T, \sigma) W(t-\sigma-\tau) d \sigma=0 \\
& \quad t-T \leq \tau \leq t
\end{aligned}
$$

which, after change of variables, gives the result.

2) Using (14) and arguing as in the derivation of (17) gives

$$
\begin{aligned}
W(\tau)+ & \Gamma B(T, T-\tau) \\
+ & \int_{0}^{T} B(T, T-\tau) W(\tau-\sigma) d \sigma=0 \\
& 0 \leq \tau \leq T .
\end{aligned}
$$

Subtracting (A.2) from (17) gives

$$
\begin{gathered}
\int_{0}^{T}[A(T, \sigma)-B(T, T-\sigma)] \\
\cdot[W(\tau-\sigma)+\Gamma \delta(\tau-\sigma)] d \sigma=0 \\
0 \leq \tau \leq T
\end{gathered}
$$

from which the result follows.
3) By differentiating (17) with respect to $T$ we obtain

$$
\begin{gathered}
\Gamma \frac{\partial A(T, \tau)}{\partial T}+A(T, T) W(T-\tau) \\
+\int_{0}^{T} \frac{\partial A(T, \sigma)}{\partial T} W(\tau-\sigma) d \sigma=0, \\
0 \leq \tau \leq T .
\end{gathered}
$$

Now, (17) may also be written (by change of variables) as

$$
\begin{gathered}
W(T-\tau)+\Gamma A(T, T-\tau) \\
+\int_{0}^{T} A(T, T-\sigma) W(\tau-\sigma) d \sigma=0 \\
\quad 0 \leq \tau \leq T .
\end{gathered}
$$

Mutiplying the above by $A(T, T)$ and subtracting the result from (A.3) gives

$$
\begin{gathered}
\int_{0}^{T}\left[\frac{\partial A(T, \sigma)}{\partial T}-A(T, T) A(T, T-\sigma)\right] \\
\cdot[W(\tau-\sigma)+\Gamma \delta(\tau-\sigma)] d \sigma=0 \\
0 \leq \tau \leq T
\end{gathered}
$$

from which the result follows.

4) By change of variable in (13) and (14) and applying Definitions (15) and (16), we obtain

$$
\begin{aligned}
& e_{f}(T, t)=y(t)+\int_{0}^{T} A(T, \sigma) y(t-\sigma) d \sigma \\
& e_{b}(T, t)=y(t-T)+\int_{0}^{T} A(T, T-\sigma) y(t-\sigma) d \sigma
\end{aligned}
$$

Differentiating (A.4) gives

$$
\begin{aligned}
\frac{\partial e_{f}(T, t)}{\partial T}= & A(T, T) y(t-T) \\
& +\int_{0}^{T} \frac{\partial A(T, \sigma)}{\partial T} y(t-\sigma) d \sigma \\
\frac{\partial e_{f}(T, t)}{\partial T}= & A(T, T) y(t-T) \\
& +A(T, T) \int_{0}^{T} A(T, T-\sigma) y(t-\sigma) d \sigma \\
= & A(T, T) e_{b}(T, t)
\end{aligned}
$$

upon substituting (19) and simplifying with (A.5). Let $\sigma^{\prime}=$ $T-\sigma$ in (A.5), then

$$
e_{b}(T, t)=y(t-T)+\int_{0}^{T} A\left(T, \sigma^{\prime}\right) y\left(t-T+\sigma^{\prime}\right) d \sigma^{\prime}
$$

from which it follows that

$$
\frac{\partial e_{b}(T, t)}{\partial T}=-\frac{\partial y(t-T)}{\partial t}+A(T, T) y(t)
$$




$$
\begin{aligned}
& -\int_{0}^{T} A\left(T, \sigma^{\prime}\right) \frac{\partial y\left(t-T+\sigma^{\prime}\right)}{\partial t} d \sigma^{\prime} \\
& +\int_{0}^{T} \frac{\partial A\left(T, \sigma^{\prime}\right)}{\partial T} y\left(t-T+\sigma^{\prime}\right) d \sigma^{\prime} \\
= & -\frac{\partial e_{b}(T, t)}{\partial t}+A(T, T) y(t) \\
& +\int_{0}^{T} \frac{\partial A(T, \sigma)}{\partial T} y(t-T+\sigma) d \sigma
\end{aligned}
$$

Applying (19) yields

$$
\begin{aligned}
\frac{\partial e_{b}(T, t)}{\partial T}= & -\frac{\partial e_{b}(T, t)}{\partial t}+A(T, T) \\
& \cdot\left[y(t)+\int_{0}^{T} A(T, T-\sigma) y(t-T+\sigma) d \sigma\right] \\
= & -\frac{\partial e_{b}(T, t)}{\partial t}+A(T, T) \\
& \cdot\left[y(t)+\int_{0}^{T} A(T, \sigma) y(t-\sigma) d \sigma\right] \\
= & -\frac{\partial e_{b}(T, t)}{\partial t}+A(T, T) e_{f}(T, t)
\end{aligned}
$$

upon simplifying using (A.4). Combining this last result and (A.6) we have the lattice structure in continuous time.

5) Using (A.5),

$$
\begin{aligned}
& \int_{0}^{T} A(\tau, \tau) e_{b}(\tau, t) d \tau \\
&=\int_{0}^{T} A(\tau, \tau) y(t-\tau) d \tau \\
&+\int_{0}^{T} \int_{0}^{\tau} A(\tau, \tau) A(\tau, \tau-\sigma) y(t-\sigma) d \sigma d \tau \\
&= \int_{0}^{T} A(\tau, \tau) y(t-\tau) d \tau \\
&+\int_{0}^{T} \int_{0}^{\tau} \frac{\partial A(\tau, \sigma)}{\partial \tau} y(t-\sigma) d \sigma d \tau \\
&= \int_{0}^{T} \frac{\partial}{\partial \tau}\left[\int_{0}^{\tau} A(\tau, \sigma) y(t-\sigma) d \sigma\right] d \tau \\
&= \int_{0}^{T} A(T, \sigma) y(t-\sigma) d \sigma \\
&=-\hat{s}\left(t \mid Y_{t-T}^{t}\right)
\end{aligned}
$$

as claimed. Equation (22) follows by a similar line of reasoning.

6) Using (A.5),

$$
\begin{aligned}
\mathcal{E}\left\{e_{b}(T, t) e_{b}(T-\tau, t)\right\} & \\
= & \mathcal{E}\left\{\left[y(t-T)+\int_{0}^{T} A(T, T-\sigma) y(t-\sigma) d \sigma\right]\right. \\
\cdot & {[y(t-T+\tau)}
\end{aligned}
$$

$$
\begin{aligned}
& \left.\left.+\int_{0}^{T-\tau} A(T-\tau, T-\tau-\sigma) y(t-\sigma) d \sigma\right]\right\} \\
= & W(\tau)+\Gamma \delta(\tau)+\int_{0}^{T} A(T, T-\sigma) \\
& \cdot[W(T-\tau-\sigma)+\Gamma \delta(T-\tau-\sigma)] d \sigma \\
& +\int_{0}^{T-\tau} A(T-\tau, T-\tau-\sigma) \\
& \cdot[W(T-\sigma)+\Gamma \delta(T-\sigma)] d \sigma \\
& +\int_{0}^{T-\tau} \int_{0}^{T} A\left(T, T-\sigma_{1}\right) A\left(T-\tau, T-\tau-\sigma_{2}\right) \\
& \cdot\left[W\left(\sigma_{1}-\sigma_{2}\right)+\Gamma \delta\left(\sigma_{1}-\sigma_{2}\right)\right] d \sigma_{1} d \sigma_{2} .
\end{aligned}
$$

By change of variables in (17),

$$
\begin{aligned}
W(\tau)+\int_{0}^{T} A(T, & T-\sigma) \\
\cdot & {[W(T-\tau-\sigma)+\Gamma \delta(T-\tau-\sigma)] d \sigma=0 }
\end{aligned}
$$

so the previous line becomes

$$
\begin{aligned}
\mathcal{E}\left\{e_{b}(T, t) e_{b}(T-\tau, t)\right\} & \\
= & \Gamma \delta(\tau)+\int_{0}^{T-\tau} A\left(T-\tau, T-\tau-\sigma_{2}\right) \\
& \cdot\left[W\left(T-\sigma_{2}\right)+\Gamma \delta\left(T-\sigma_{2}\right)\right] d \sigma_{2} \\
+ & \int_{0}^{T-\tau} \int_{0}^{T} A\left(T, T-\sigma_{1}\right) A\left(T-\tau, T-\tau-\sigma_{2}\right) \\
& \cdot\left[W\left(\sigma_{1}-\sigma_{2}\right)+\Gamma \delta\left(\sigma_{1}-\sigma_{2}\right)\right] d \sigma_{1} d \sigma_{2} \\
= & \Gamma \delta(\tau)+\int_{0}^{T-\tau} A\left(T-\tau, T-\tau-\sigma_{2}\right) \\
& \cdot\left[\Gamma \delta\left(T-\sigma_{2}\right)+W\left(T-\sigma_{2}\right)+\int_{0}^{T} A\left(T, T-\sigma_{1}\right)\right. \\
& \left.\cdot\left[W\left(\sigma_{1}-\sigma_{2}\right)+\Gamma \delta\left(\sigma_{1}-\sigma_{2}\right)\right] d \sigma_{1}\right] d \sigma_{2} .
\end{aligned}
$$

By change of variables this can be written as

$$
\begin{aligned}
\mathcal{E}\left\{e_{b}(T, t) e_{b}(T-\tau, t)\right\} & \\
= & \Gamma \delta(\tau)+\int_{0}^{T-\tau} A\left(T-\tau, T-\tau-\sigma_{2}\right) \\
\cdot & {\left[\Gamma \delta\left(T-\sigma_{2}\right)+W\left(T-\sigma_{2}\right)+\int_{0}^{T} A\left(T, \sigma_{1}\right)\right.} \\
\cdot & {\left.\left[W\left(T-\sigma_{1}-\sigma_{2}\right)+\Gamma \delta\left(T-\sigma_{1}-\sigma_{2}\right)\right] d \sigma_{1}\right] d \sigma_{2} }
\end{aligned}
$$

and by application of (A.1), this can be further simplified to

$$
\begin{aligned}
& \mathcal{E}\left\{e_{b}(T, t) e_{b}(T-\tau, t)\right\} \\
& \quad=\Gamma \delta(\tau)+\int_{0}^{T-\tau} A\left(T-\tau, T-\tau-\sigma_{2}\right) \Gamma \delta\left(T-\sigma_{2}\right) d \sigma_{2} \\
& \quad=0, \quad \tau>0 .
\end{aligned}
$$

Equation (24) follows by a similar line of reasoning. 


\section{REFERENCES}

[1] L. G. Chalmers, Integral Equations: A Short Course. London, United Kingdom: International Textbook Company, 1976.

[2] P. Dewilde, A. C. Vieira, and T. Kailath, "On an generalized SzegoLevinson realization algorithm for optimal linear predictors based on a network synthesis approach," IEEE Trans. Circuits Syst., vol. CAS-25, pp. 663-675, Sept. 1978 .

[3] M. R. Gevers and V. J. Wertz, "A d-step predictor in lattice and ladder form," IEEE Trans. Automat. Contr., vol. AC-28, pp. 465-476, Apr. 1983 .

[4] G. C. Goodwin, R. H. Middleton, and H. V. Poor, "High-speed digital signal processing and control," Proc. IEEE, vol. 80, pp. 240-259, Feb. 1992.

[5] G. C. Goodwin and K. S. Sin, Adaptive Filtering, Prediction and Control. Englewood Cliffs, NJ: Prentice-Hall, 1983.

[6] M. L. Honig and D. G. Messerschmitt, Adaptive Filters: Structures Algorithms and Applications. Boston: Kluwer, 1984.

[7] F. Itakura and S. Saito, "Digital filtering techniques for speech analysis and synthesis," in Proc. 7th Int. Congress Acoust., 1971.

[8] F. Jabbari, "Lattice filters for RLS estimation of a delta operator based model, Tech. Rep., Dept. of Mechanical Engineering, Univ. California Irvine, 1990.

[9] T. Kailath, "A view of three decades of linear filtering theory," IEEE Trans. Inform. Theory, vol. IT-20, pp. 146-181, Mar. 1974.

[10] T. Kailath, B. C. Levy, L. Ljung, and M. Morf, "Fast time-invarian implementations of gaussian signal detectors," IEEE Trans. Inform. Theory, vol. IT-24, pp. 469-477, July 1978.

[11] S. K. Kay and S. L. Marple, Jr., Spectrum analysis-A modern perspective," Proc. IEEE, vol. 69, pp. 1380-1419, Nov. 1981.

[12] A. Le Breton and D. T. Pham, "Maximum likelihood estimate for continuous time autoregressive models and applications," Tech. Rep., Univ. Grenoble, 1990.

[13] B. Levy, Kailath T., L. Ljung, and M. Morf, "Fast time-invarian implementations for linear least squares smoothing filters," IEEE Trans. Automat. Contr., vol. AC-24, pp. 770-774, Oct. 1979.

[14] D. G. Luenberger, Optimization by Vector Space Methods. New York: Wiley, 1969.

[15] R. H. Middleton and G. C. Goodwin, Digital Control and Estimation-A Unified Approach. Englewood Cliffs, NJ: Prentice-Hall, 1990.

[16] M. Morf, D. T. Lee, J. R. Nicholls, and A. Vieira, "A classification of algorithms for ARMA models and ladder realizations," in Proc. 1977 IEEE Int. Conf. Acoust., Speech, Signal Processing, pp. 13-19, June 1977.

[17] D. T. Pham and A. Le Breton, "Levinson-Durbin type algorithms for continuous time autoregressive models and applications," Math. Contr., Signals, Syst., vol. 4, pp. 69-79, Apr. 1991.

[18] V. Vijayan, H. V. Poor, J. B. Moore, and G. C. Goodwin, "A Levinsontype algorithm for modeling fast-sampled data," IEEE Trans. Automat. Contr., vol. AC-36, pp. 314-321, Mar. 1991.

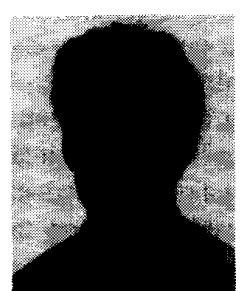

Steven R. Weller received the B.E. (Hons I) degre in computer engineering in 1988, and the M.E. degree in electrical engineering in 1992, both from the University of Newcastle, New South Wales, Australia.

From 1988 to 1990 he worked as an engineer with the Centre for Industrial Control Science, Newcastle, Australia. He is currently a candidate for the Ph.D. degree in electrical engineering at the University of Newcastle. His current research interests are in multivariable control theory and its applications, adaptive control, and linear filtering theory.

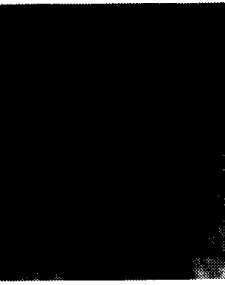

Arie Feuer received the B.Sc. and M.Sc. degrees from the Technion, Haifa, Israel in 1967 and 1973, respectively, and the Ph.D. degree from Yale University, New Haven, CT, in 1978.

From 1967 to 1970 he was employed by Technomtic-Israel, working on factory automation. From 1978 through 1983 with Bell Laboratories, Holmdel, NJ, studying telephone network performance. Since 1983, he has been on the faculty of the Electrical Engineering Department, Technion, Haifa, Israel. His research interests are in adaptive control, adaptive signal processing, and hybrid systems.

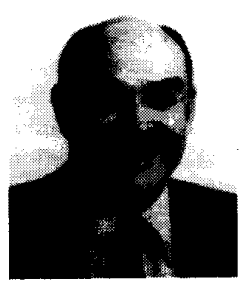

Graham C. Goodwin received the B.Sc (Physics), B.E (Electrical Engineering), and Ph.D from the University of New South Wales.

From 1970 until 1974 he was a lecturer in the Department of Computing and Control, Imperial College, London, Since 1974 he has been with the Department of Electrical Engineering and Computer Science, University of Newcastle, Australia. He is currently Professor of Electrical Engineering and Director of the Centre for Industrial Control Science at the University of Newcastle. He is the co-author of four books: Control Theory (Oliver and Boyd 1970), Dynamic System Identification (Academic Press 1977), Adaptive Filtering, Prediction and Control. (Prentice Hall 1984), and Digital Control and Estimation (Prentice Hall 1989.

Dr. Goodwin is the recipient of several international prizes including a best paper award by IEEE TRANS. AuTOMATIC CONTROL, and best engineering text book award from the International Federation of Automatic Control. Graham Goodwin is a Fellow of the Australian Academy of Technology, Science and Engineering.

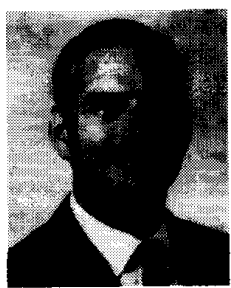

H. Vincent Poor (S'72-M'77-SM'82-F'87) graduated with highest honors from Auburn University in 1972, and received the Ph.D. degree in Electrical Engineering and Computer Science from Princeton University in 1977.

In that year he joined the University of Illinois, where, from 1984, he was Professor of Electrical and Computer Engineering, and Research Professor in the Coordinated Science Laboratory. In 1990, he joined Princeton University where he is currently Professor of Electrical Engineering. He has also held visiting appointments at Imperial College (London) and at the University of Newcastle (Australia). Dr. Poor's research interests are in the area of statistical signal processing in communications and control, and his publications in this area include the textbook An Introduction to Signal Detection and Estimation. $\mathrm{He}$ is a member of the editorial boards of several journals, including Mathematics of Control, Signals and Systems; the International Journal of Imaging Systems and Technology; and the International Journal of Robust and Nonlinear Control.

Dr. Poor is a Fellow of the American Association for the Advancement of Science; a Fellow of the Acoustical Society of America; and a member of the Institute of Mathematical Statistics. He has been involved in a number of IEEE activities, including as an Associate Editor of the IEEE TRANSACTIONS ON AUTOMATIC CONTROL and of the IEEE TRANSACTIONS ON INFORMATION THEORY; as Program Chair for the 25th IEEE Conference on Decision and Control (1986); as General Chair for the 1989 American Control Conference; as 1990 President of the IEEE Information Theory Society; and as a member of the IEEE Board of Directors from 1991-1992. He is currently serving on the Board of Governors of the IEEE Control Systems Society. In 1992, Dr. Poor received the Frederick Emmons Terman Award from the American Society for Engineering Education. 